\title{
Political Biases in Lobbying under Asymmetric Information ${ }^{1}$
}

\author{
David Martimort ${ }^{2}$ and Aggey Semenov ${ }^{3}$
}

This version: 6th September 2006

\begin{abstract}
This paper introduces asymmetric information in a pluralistic model of interest groups competition and analyzes its impact on policy biases. Lobbying groups are uninformed on a decision-maker's preferences and use nonlinear contributions not only to compete for the agent's services but also to learn about his preferences in an otherwise standard common agency model of lobbying. Asymmetric information can be, either on the decision-maker's ideal point (horizontal differentiation) or on the strength of his own preferences for ideology (vertical differentiation). At equilibrium, asymmetric information redistributes bargaining powers between interest groups and the decision-maker in non-trivial ways that may depend on the kind of informational asymmetry which is postulated. Asymmetric information tends to mitigate the influence of interest groups and contributions might be significantly reduced. Interest groups no longer contribute for a change in policy what it is worth to them as under complete information. Contributions incorporate a discount related to the group's ability to solve the asymmetric information problem.
\end{abstract}

Keywords: Common Agency, Lobbying Competition, Asymmetric Information.

JEL Classification : D72; D82.

\section{Introduction}

Two different and somewhat complementary views of how interest groups (thereafter IGs) influence policy-making prevail nowadays in the economic literature. The first approach follows what political scientists would coin as being the pluralistic view of politics. ${ }^{4}$ This trend of research assumes a priori that some IGs (which have already succeeded in solv-

\footnotetext{
${ }^{1}$ This paper was prepared for the EEA 2006 Meeting in Vienna. We thank seminar participants there. The usual disclaimer applies.

${ }^{2}$ University of Toulouse, Institut Universitaire de France and EHESS.

${ }^{3}$ National University of Singapore.

${ }^{4}$ See Dahl (1961).
} 
ing the Olsonian collective action curse) are involved in a hidden gaming whose resulting equilibrium reflects the power of those existing political forces. Even though earlier contributions in this line of thought can be traced back to Bentley (1908), it is more deeply associated to the Chicago School $^{5}$ and certainly culminated more recently with the work of Grossman et Helpman (1994) among others. These authors viewed the political game played by IGs as a common agency game. ${ }^{6}$ IGs (the principals) influence a decision-maker (the common agent) by means of contributions which depend on the policy that the latter chooses. For the so-called "truthful" equilibria of these complete information games, each IG's marginal contribution for a policy change reflects his preferences among alternatives. The resulting equilibrium policy is thus efficient, i.e., it maximizes the aggregate payoff of the grand-coalition formed of all the existing groups and the decision-maker. The distribution of the political surplus reflects the political power of the different organized groups and how the decision-maker can use lobbying competition to pit one group against the other. In the complete information environment, the power of a group can be roughly defined as its ability to move policy towards his own ideal point.

The second approach of how interest groups influence policy relies instead on private information as the engine of influence on the political process. There is no room here to give full credit to all contributions which describe this informational channel. Grossman and Helpman (2001) offer an exhaustive presentation of the issues that arises in signalling models where informed IGs move first and influence decision-makers only by means of communication. The New Regulatory Economics ${ }^{7}$ puts also informational asymmetries and the resulting trade-off between allocative efficiency and rent extraction at the core of the analysis but stresses also that monetary transfers can be used to mitigate informational conflicts. Regulatory institutions and contracts respond then to the influence of IGs and aim at limiting the informational rents that those groups might withdraw from public decision-making. In this approach, the political power of a group is related to its ability to secure some informational rent. Indeed, it is a basic lesson of Incentive Theory that asymmetric information might introduce some bias towards the ideal point of the informed party. $^{8}$ Although it helps justifying the forces that create IGs in the first place, this informational approach somewhat neglects competition among IGs and, by focusing on an ex ante optimal design of institutions and contracts, it might be viewed by some scholars as giving too much commitment power to decision-makers. In other words and to summarize roughly, the weakness of the pluralistic approach corresponds to the strength of the informational approach and vice versa.

There is certainly still a long way before fully reconciling those two views of the

\footnotetext{
${ }^{5}$ See Peltzman (1976) and Becker (1983) among others.

${ }^{6}$ See Bernheim and Whinston (1986) for a presentation of those games.

${ }^{7}$ See Laffont and Tirole (1993) among others.

${ }^{8}$ See Laffont and Martimort (2002) for instance.
} 
political process and having a model which simultaneously explains institutional design and endogenizes groups formation and political biases. ${ }^{9}$ Although it goes towards this direction, this short paper should only be viewed as a first exploratory step on this avenue. In the sequel, we introduce various forms of asymmetric information in an otherwise standard common agency model of pluralistic politics. The IGs' monetary contributions not only have an influence role but are also used as screening devices. This extension of the basic pluralistic model yields the following insights:

- First, asymmetric information redistributes bargaining powers among IGs and decisionmakers in non-trivial ways. As such, asymmetric information is an important ingredient to explain both some systematic biases towards either some IGs or the decision-maker.

- Second, under asymmetric information, IGs no longer contribute for a policy change what it is worth to them as under complete information but contributions incorporate a discount related to their ability to solve the asymmetric information problem. This discount might be so large that some groups may prefer to eschew intervention on DMs who are too hard to influence. Different groups do not suffer in the same way from paying this discount and this is reflected in their resulting influence on the political process. ${ }^{10}$

Section 2 presents the model. In Section 3, we introduce some form of vertical differentiation. DMs trade off social welfare maximization against the monetary contributions they receive from IGs. They have the same ideal policy but differ in terms of the weight they give to ideology which is private information. IGs ignore this weight. The equilibrium policy may be systematically biased towards the weakest $I G$, the one whose preferences are further away from the decision-maker is the policy space. In Section 4, we analyze the case of horizontal differentiation. Decision-makers differ now in terms of their most preferred policy and have private information on this parameter. The equilibrium policy might be systematically biased towards the decision-maker's ideal point featuring some fundamental gridlock and status quo bias of economic policy. Contributions are small, sometimes even null when horizontal uncertainty is large enough. Section 5 offers some synthetic perspective on the forces that affect political biases under asymmetric information. ${ }^{11}$

\footnotetext{
${ }^{9}$ The ultimate objective of such analysis should be to predict under which legislative organization some interest groups may be more efficient at intervening than others.

${ }^{10}$ Using a complete information model of lobbying in the context of import tariffs for intermediate goods, Gawande and Krishna (2005) argued for instance that the competition between IGs (final producers and intermediate ones) whose impacts cancel out might explain that the estimated implicit weight given to contributions in the decision-maker's preferences is rather low. Asymmetric information would magnify this bias towards the decision-maker's ideal point.

${ }^{11}$ Proofs are relegated to an Appendix.
} 


\section{The Model}

Two IGs (also called principals) $P_{1}$ and $P_{2}$ influence a decision-maker (the agent, thereafter DM) who chooses a policy $q$ in a one dimensional space. $P_{1}$ has an ideal point located at $a_{1}=a+b$, whereas $P_{2}$ 's ideal point is located at $a_{2}=-a{ }^{12}$ The DM's ideal point is at $\theta$. This ideal point can be viewed as a socially optimal policy when both the well-beings of unorganized and organized IGs are taken into account. It can also be viewed as the median voter's ideal point if the DM's objectives are aligned with those of voters as a whole or as an ideological status quo otherwise. The IGs and the DM have quasi-linear utility functions respectively given by

$$
V_{i}=-\frac{1}{2}\left(q-a_{i}\right)^{2}-t_{i}, \quad \text { for } i=1,2 \text { and } \quad U=-\frac{\beta}{2}(q-\theta)^{2}+t_{1}+t_{2},
$$

where $\beta$ is a scale parameter capturing the intensity of DM's ideological preferences towards his own ideal point. Contributions $t_{i}$ are non-negative.

Under horizontal asymmetric information, DMs differ in terms of their ideal points $\theta .^{13}$ Under vertical asymmetric information, DMs have the same ideal point but differ in terms of their ideological bias $\beta .^{14}$

Timing: The lobbying game unfolds as follows. First, both IGs offer non-cooperatively and commit to contribution schedules $t_{i}(q)$ which stipulate a monetary transfer depending on the realized policy. Second, the DM decides whose offers to accept (if any) and then chooses an optimal policy.

Complete Information: The efficient policy $q^{*}(\theta, \beta)$ which maximizes the aggregate payoff of the grand-coalition formed by both IGs and the DM is a weighted average of the different player's ideal points with weights reflecting their ideological biases:

$$
q^{*}(\theta, \beta)=\frac{b+\beta \theta}{2+\beta} .
$$

When $b>0$, this policy is biased towards $P_{1}$ 's ideal point which is further away from the DM's own ideal point than $P_{2}$ 's. Henceforth, we will refer to $P_{1}$ (resp. $P_{2}$ ) as the weak (resp. strong) principal. Moreover, as $\beta$ increases, $q^{*}(\theta, \beta)$ comes closer to the DM's ideal point reflecting the compromising role of DM's ideology. From Bernheim and

\footnotetext{
${ }^{12}$ We assume $a>0$ and $b \geq 0$.

${ }^{13}$ That IGs are uninformed on this parameter can be justified when there exists a number of constraints on the political process that are ignored by those groups. Typical examples would be the slack on the budget constraint faced by the decision-maker or the strength of unmodeled IGs' influence. Even when individuals have been elected on the basis of some ideological platforms, there still remains considerable uncertainty on their incentives to stick to this platform ex post. To quote Charles Pasqua (a former French Minister), "Promises only commit those who listen to them"...

${ }^{14}$ Typically, IGs may have difficulties in figuring out the importance of ideology for a newly appointed decision-maker.
} 
Whinston (1986), this first-best outcome can be implemented through truthful schedules $t_{i}(q)=\max \left\{0,-\frac{1}{2}\left(q-a_{i}\right)^{2}-C_{i}\right\} i=1,2$ for some constants $\left(C_{1}, C_{2}\right)$ which actually give the IGs' equilibrium payoffs. ${ }^{15}$

\section{Vertical Asymmetric Information}

The DM has now private information on $\beta$ but this ideological bias is not too strong; $\beta$ is uniformly distributed on $[0, \bar{\beta}]$ for $\bar{\beta} \leq 1$. For simplicity, we also assume that $\theta=0$.

Proposition 1 Assume vertical asymmetric information on the DM's preferences. Then the equilibrium policy $q^{e}(\beta)$ is upward distorted w.r.t. first-best $q^{*}(0, \beta)$ :

$$
q^{e}(\beta)=\frac{b}{2+3 \beta-2 \bar{\beta}} \geq q^{*}(0, \beta) \text { with equality only at } \beta=\bar{\beta} .
$$

The DM always gets a strictly positive payoff in equilibrium. The weak principal's marginal contribution is greater than that of the strong principal.

Each IG wants of course to push the policy towards his own ideal point. Absent the other group's intervention, this requires a greater (resp. lower) transfer when the DM has a strong (resp. weak) ideological bias. Under asymmetric information and still absent the other group's intervention, a DM with a low ideological bias would thus be tempted to exaggerate this bias to receive greater compensations from that group. ${ }^{16}$ This would call for leaving a positive information rent to DMs having small ideological biases. When IGs bid instead for favors, both offer large contributions to those DMs. DMs with stronger ideological biases find it now attractive to pretend having less. ${ }^{17}$ Because IGs have opposite preferences, each of those can only mitigate the equilibrium policy that the other would induce being alone. This makes this policy less sensitive to the DM's ideological bias. In other words, the information rent that this DM gets by taking both contracts is less steep than when taking only one of those and only the $\beta=0 \mathrm{DM}$ is indifferent between taking both contributions or only one. To limit the extra rent left to DMs with stronger ideological biases, both IGs offer contributions which have less mitigating power compared with what they offer when knowing $\beta$. This is true for both principals but the strong one is doing so even more. Indeed, contributions are designed to counter the other IG's preferences and the weak IG's marginal contribution is greater because the strong

\footnotetext{
${ }^{15}$ The constants $\left(C_{1}, C_{2}\right)$ are obtained by solving a system of equations coming from specifying the DM's participation constraints: $\sum_{i=1}^{2} t_{i}\left(q^{*}(\theta, \beta)\right)-\frac{\beta}{2}\left(q^{*}(\theta, \beta)-\theta\right)^{2}=\max \left\{0, \max _{q} t_{i}(q)-\frac{\beta}{2}(q-\theta)^{2}\right\}$. See also Laussel and Lebreton (2001) on that.

${ }^{16}$ Incentive constraints are binding upward.

${ }^{17}$ Incentive constraints are now binding downwards.
} 
one is close to the DM in the policy space. At equilibrium, the equilibrium policy is thus upward distorted for all types $\beta<\bar{\beta}$. ${ }^{18}$ Vertical asymmetric information redistributes somewhat the bargaining power in favor of the weakest IG. ${ }^{19,20}$

\section{Horizontal Asymmetric Information}

Let us now turn to the case where $\theta$ is private information, drawn uniformly on an interval $[-\delta, \delta]$. To simplify, both IGs are symmetrically located around 0 , i.e., $b=0$. For the purpose of this paper, we focus on $\beta>1$, i.e., the DM's ideological preferences are now sufficiently pronounced.

To understand the impact of horizontal asymmetric information, it is first useful to think of $P_{1}$ as being the only IG around. For the moment, let us assume $a>\delta$, i.e., whatever the agent's ideal point, the IG's preference is more extreme. Under complete information, $P_{1}$ would offer a policy maximizing the aggregate payoff of the bilateral coalition formed with the agent, i.e., $q_{1}^{*}(\theta)=\frac{a+\beta \theta}{1+\beta}$. When $a>\delta$, this policy is always greater than $\theta$, i.e., unambiguously biased in the direction of $P_{1}$ 's ideal point. Under complete information, inducing the agent to adopt this policy requires giving to the DM a reward $t_{1}^{*}(\theta)=\frac{\beta}{2(1+\beta)^{2}}(a-\theta)^{2}$ leaving him just indifferent between the status quo policy $\theta$ and choosing $q_{1}^{*}(\theta)$. Of course, this reward is greater for more moderate types.

Under asymmetric information, such scheme is not incentive compatible. More extreme types who are closer to $P_{1}$ 's own ideal point would like to appear moderate to grasp those high transfers. To avoid this, $P_{1}$ increases the distance between the policy suggested to moderate types and his own ideal point. Reducing the information rent of extremist DMs calls for distorting the policy in the direction of the agent's ideal point and paying less transfer to moderate types. However, $P_{1}$ is constrained in doing so by the fact that the DM may always refuse any contribution and choose the status quo policy.

\footnotetext{
${ }^{18}$ At equilibrium, everything happens as if the true ideological bias $\beta$ was replaced by a lower virtual bias $\tilde{\beta}=3 \beta-2 \bar{\beta} \leq \beta$.

${ }^{19}$ Lebreton and Salanié (2003) analyzed a model with the similar features although the sets of possible policies and contributions are finite. Suppose that the DM has to choose between the IGs' ideal points. Efficiency from the grand-coalition's viewpoint calls for choosing $-a$ since $P_{2}$ 's ideal point is closer to the agent's one. Under asymmetric information, assume that $P_{i}$ only makes a contribution $t_{i}$ when $a_{i}$ is chosen. Compared with our framework which entails overlapping areas of influence, the lobbying game is turned into a pure head-to-head competition. The inefficient policy $a+b$ is chosen by the DM whenever $t_{1}-\frac{\beta}{2}(a+b)^{2} \geq t_{2}-\frac{\beta}{2} a^{2}$ i.e., when $\beta \leq \frac{2\left(t_{1}-t_{2}\right)}{b(2 a+b)}=\beta^{*}$. The equilibrium contributions are respectively $\frac{1}{2}(2 a+b)^{2}-t_{1}^{e}=\frac{b(2 a+b)}{2} \beta^{*}$ and $\frac{1}{2}(2 a+b)^{2}-t_{2}^{e}=\frac{b(2 a+b)}{2}\left(\bar{\beta}-\beta^{*}\right)$. From this, we obtain $\beta^{*}=\frac{\bar{\beta}}{3}$ and $t_{1}^{e}>t_{2}^{e}$. The equilibrium under asymmetric information is inefficient with the weak principal $P_{1}$ 's ideal point being now chosen when $\beta \leq \beta^{*}$.

${ }^{20}$ The fact that the IGs are asymmetric (i.e., $b>0$ ) is crucial. Otherwise, the first-best efficient policy would always be the agent's ideal point even under asymmetric information (see (1)) and asymmetric information induces no extra bias.
} 
The second-best optimal policy is now defined as:

$$
q_{1}^{e}(\theta)=\max \left\{\frac{a+\beta(2 \theta-\delta)}{1+\beta}, \theta\right\} \leq q_{1}^{*}(\theta) .
$$

The optimal policy in this monopolistic screening environment is thus at least the agent's ideal point. It might be distorted upward towards the IG's ideal point only over the interval $\left[\frac{\beta \delta-a}{\beta-1}, \delta\right]$. When $a \leq \delta$, this interval is empty. Hence, the best-response of $P_{1}$ to a null contribution by $P_{2}$ is itself also null.

Proposition 2 Assume horizontal asymmetric information on the DM's ideal point with $a \leq \delta$ and $\beta>1$. Then, there exists an equilibrium where IGs do not contribute and the status quo policy $q^{e}(\theta)=\theta$ is implemented.

Asymmetric information undermines significantly the influence of IGs as soon as horizontal uncertainty is large enough. It is akin to an implicit increase of the DM's bargaining power in the political process. Because they are symmetrically located around the agent's expected ideal point, none of the IGs gains anything from this bias contrary to the case of vertical uncertainty.

Under horizontal asymmetric information, different kinds of equilibria may emerge depending on whether the DM is easily influenced or not and depending on the uncertainty on his preferences. Martimort and Semenov (2006) describe such equilibria and show that, as uncertainty on the agent's type diminishes, IGs may first secure areas with unchallenged influence, then contributions may overlap for the most moderate types and finally for all types if uncertainty is very small. The fact that contributions may be null over some range provides a nice endogenous reasons for why a group fails to intervene on ideologically too distant DMs.

\section{Concluding Remarks}

Although they significantly differ in terms of the directions of political biases induced by asymmetric information, both models above have some common features that we would like to stress in this concluding section. In both cases, the DM's type whose allocation attracts other types would receive the greater contributions had contracting taken place under complete information. This may be due to the fact that this DM is easily influenced and IGs competition increases contributions in the first model. This may instead be due to the fact that the DM is too moderate and hard to move away towards the more extremist views of IGs in the second model even if those groups secure unchallenged influence. Asymmetric information and the desire of IGs to reduce the DM's information rent calls 
for mitigating the role of IGs, making them less willing to contribute and to influence those types who are so attractive for others. In both cases, the equilibrium political bias that results reflects who are the "winners" of the game under asymmetric information, i.e., those groups or decision-makers who suffer less from it, but in any case, IGs find influence a much harder job.

\section{References}

Becker, G., (1983), "A Theory of Competition Among Pressure Groups for Political Influence", Quarterly Journal of Economics, 98: 371-400.

Bentley, A., (1908), The Process of Government, Chicago: Chicago University Press.

Bernheim, D. and M. Whinston, (1986), "Menu Auctions, Resource Allocations and Economic Influence", Quarterly Journal of Economics, 101: 1-31.

Dahl, R., (1961), Who Governs? Democracy and Power in American City, New Haven: Yale University Press.

Gawande, K. and P. Krishna, (2005), "Lobbying Competition over U.S. Trade Policy", NBER Working Paper No 11371.

Grossman, G. and E. Helpman, (1994), "Protection for Sale", American Economic Review, 84: $833-850$.

Grossman, G. and E. Helpman, (2001), Special Interest Politics, Cambridge: MIT Press.

Laffont, J.J. and D. Martimort, (2002), The Theory of Incentives: The Principal-Agent Model, Princeton University Press, Princeton.

Laffont, J.J. and J. Tirole, (1993), A Theory of Incentives in Regulation and Procurement, MIT Press, Cambridge.

Laussel, D. and M. Lebreton, (2001), "Conflict and Cooperation: The Structure of Equilibrium Payoffs in Common Agency", Journal of Economic Theory, 100: 93-128.

Lebreton, M. and F. Salanié, (2003), "Lobbying under Political Uncertainty", Journal of Public Economics, 87: 2589-2610.

Martimort, D. and A. Semenov, (2006), "Ideological Uncertainty and Lobbying Competition", mimeo IDEI Toulouse.

Martimort, D. and L. Stole, (2005), "On the Robustness of Truthful Equilibria in Common Agency Games", mimeo IDEI Toulouse and University of Chicago.

Peltzman, S., (1976), "Towards a More General Theory of Regulation", Journal of Law 
and Economics, 19: 211-240.

Seierstad, A. and K. Sydsaeter, (1987), Optimal Control Theory with Economic Applications, Amsterdam, North Holland.

\section{Appendix}

- Proof of Proposition 1: We suppose that $P_{-i}$ offers a non-negative contribution $t_{-i}(q) \geq 0$ and look for $P_{i}$ 's best-response. For ease of notations, denote respectively $U_{i}(\beta)=\max _{q} t_{i}(q)-\frac{\beta}{2} q^{2}$ and $U(\beta)=\max _{q} t_{1}(q)+t_{2}(q)-\frac{\beta}{2} q^{2}$ the agent's non-negative information rent when taking only $P_{i}$ 's contribution and when taking both. Incentive compatibility yields at any point of differentiability:

$$
\dot{U}(\beta)=-\frac{q^{2}(\beta)}{2} \text { with } \ddot{U}(\beta) \geq 0
$$

and $\dot{U}_{i}(\beta)=-\frac{q_{i}^{2}(\beta)}{2}$ with $\ddot{U}_{i}(\beta) \geq 0$ where $q(\beta)=\arg \max _{q} t_{1}(q)+t_{2}(q)-\frac{\beta}{2} q^{2}$ and $q_{i}(\beta)=$ $\arg \max _{q} t_{i}(q)-\frac{\beta}{2} q^{2}$. $P_{i}$ 's best-response is obtained when solving

$$
\begin{aligned}
\left(\mathcal{P}_{i}\right): \max _{\{q(\cdot), U(\cdot)\}} \int_{0}^{\bar{\beta}}\left(-\frac{1}{2}\left(q(\beta)-a_{i}\right)^{2}-\frac{\beta}{2} q^{2}(\beta)+t_{-i}(q(\beta))-U(\beta)\right) d \beta \\
\quad \text { subject to }(3) \text { and } U(\beta) \geq U_{-i}(\beta) \geq 0, \quad \forall \beta \in[0, \bar{\beta}] .
\end{aligned}
$$

As soon as $\left|q_{-i}(\beta)\right|>|q(\beta)| \quad \forall \beta \in[0, \bar{\beta}]$ (a condition to be checked ex post), the participation constraint (4) is binding only at $\beta=0$. Using $U(\beta)=U_{-i}(0)-\int_{0}^{\beta} \frac{q^{2}(x)}{2} d x$, inserting this expression into the maximand of $\left(\mathcal{P}_{i}\right)$, integrating by parts and, finally optimizing pointwise leads to the following necessary FOC: ${ }^{21}$

$$
-\left(q(\beta)-a_{i}\right)-\beta q(\beta)+t_{-i}^{\prime}(q(\beta))=(\beta-\bar{\beta}) q(\beta) .
$$

Summing those conditions over $i$ and taking into account that the agent's choice satisfies $\sum_{i=1}^{2} t_{i}^{\prime}(q(\beta))=\beta q(\beta)$ yields the equilibrium policy $q^{e}(\beta)$ given in (1). It is decreasing and positive as requested by the SOC in (3). Replacing $\beta$ by its expression in terms of $q$ obtained from (1) into (5) yields $P_{i}$ 's marginal contribution for any $q$ in the range of $q^{e}(\cdot)$ :

$$
t_{i}^{\prime}(q)=-\frac{(1-\bar{\beta})}{3} q-a_{-i}+\frac{2}{3} b, \quad \forall i \in\{1,2\} .
$$

These schedules are concave as soon as $\bar{\beta} \leq 1$ as assumed. Following Martimort and Stole (2005), the expression (6) will be extended for any output as long as $t_{i}(q) \geq 0$. From (6), we deduce immediately the expression of the policy had the DM refused $P_{i}$ 's

\footnotetext{
${ }^{21}$ It is also sufficient by concavity of the objective as it can be checked ex post once the expression of the equilibrium schedule $t_{-i}(\cdot)$ is derived.
} 
contribution: $q_{i}(\beta)=\frac{2 b-3 a_{-i}}{3 \beta+1-\beta}$ as long as $t_{i}\left(q_{i}(\beta)\right) \geq 0 .{ }^{22}$ Integrating (6), we obtain the nonlinear equilibrium schedules as

$$
t_{i}(q)=\max \left\{0,-\frac{(1-\bar{\beta})}{6} q^{2}-\left(a_{-i}-\frac{2}{3} b\right) q-C_{i}\right\}, \quad \forall i \in\{1,2\} .
$$

Observe that $t_{1}^{\prime}(q)>t_{2}^{\prime}(q)$, capturing the fact that the weak principal contributes more at the margin than the strongest one's. The constants $\left(C_{1}, C_{2}\right)$ are then determined by the binding participation constraints at $0: U(0)=U_{1}(0)=U_{2}(0)>0$ which yields $C_{1}=-\frac{18 a^{2}+12 a b+7 b^{2}}{12(1-\bar{\beta})}$ and $C_{2}=-\frac{18 a^{2}+24 a b+b^{2}}{12(1-\bar{\beta})}$. With those expressions of $\left(C_{1}, C_{2}\right)$, one can easily check that the agent always gets a positive rent for any $\beta$.

- Proof of Proposition 2: Denote $U(\theta)=\max _{q} t_{1}(q)-\frac{\beta}{2}(q-\theta)^{2}$, DM's non-negative information rent. Incentive compatibility implies at any point of differentiability:

$$
\dot{U}(\theta)=\beta(q(\theta)-\theta) \text { with } \ddot{U}(\beta) \geq 0
$$

where $q(\theta)=\arg \max _{q} t_{1}(q)-\frac{\beta}{2}(q-\theta)^{2}$. $P_{1}$ 's best-response to a null contribution offered by $P_{2}$ is thus obtained when solving:

$$
\begin{gathered}
\left(\mathcal{P}_{1}\right): \max _{\{q(\cdot), U(\cdot)\}} \int_{-\delta}^{\delta}\left(-\frac{1}{2}(q(\theta)-a)^{2}-\frac{\beta}{2}(q(\theta)-\theta)^{2}-U(\theta)\right) d \theta \\
\quad \text { subject to }(8) \text { and } U(\theta) \geq 0 \quad \forall \theta \in[-\delta, \delta] .
\end{gathered}
$$

The Lagrangean for this optimization problem can be written as:

$$
L(q, U, \lambda, \mu)=-\frac{1}{2}(q-a)^{2}-\frac{\beta}{2}(q-\theta)^{2}-U+\lambda \beta(q-\theta)+\mu U
$$

where $\lambda$ is the costate variable and $\mu \geq 0$ is the Lagrange multiplier of the pure state constraint (9). Using the necessary and sufficient conditions for optimality, ${ }^{23}$ we find $\dot{\lambda}(\theta)=1-\mu(\theta)$ and thus $\lambda(\theta)=\theta-\delta-M(\theta)$ for some non-decreasing function $M(\cdot)$ such that $M(\delta)=0$. Optimizing pointwise w.r.t. $q(\cdot)$ yields:

$$
-(q(\theta)-a)-\beta(q(\theta)-\theta)+\beta(\theta-\delta-M(\theta))=0 .
$$

We can now easily guess the candidate solution: $\mu(\theta)=\frac{\beta-1}{\beta}>0$ (resp. 0) on $\left[-\delta, \frac{\beta \delta-a}{\beta-1}\right]$ where $q^{e}(\theta)=\theta\left(\operatorname{resp} .\left[\frac{\beta \delta-a}{\beta-1}, \delta\right]\right.$ where $\left.q^{e}(\theta)=\frac{a+\beta(2 \theta-\delta)}{1+\beta}\right)$.

\footnotetext{
${ }^{22}$ Note that $\dot{q}_{i}(\beta)<0$ as requested by the second-order condition. It is also easily checked that $\left|q_{-i}(\beta)\right|>|q(\beta)| \quad \forall \beta \in[0, \bar{\beta}]$ so that (4) binds indeed only at 0 and is slack everywhere else.

${ }^{23}$ See Seierstad and Sydsaeter (1987, Theorem 1, p. 317-319).
} 